\title{
Texture Analysis Using Multifractal Spectrum
}

\author{
Khaled Harrar and Mohamed Khider
}

\begin{abstract}
Multifractal analysis has been recognized as a powerful tool in characterizing textures. Several studies have shown the possibilities offered by multifractal analysis in image processing, in particular in classification of complex textures. Indeed, in most cases, the mode of multifractal spectrum is used for classification; in this study, we propose two different methods to estimate this spectrum. This paper focuses on the classification of Brodatz textures using multifractal analysis. Two methods are considered: The first method is based on the multifractal formalism of Frish and Parisi through the Legendre transform, the second one is a direct method based on the box-counting algorithm. For both approaches, we used the multiresolution coefficients of the wavelet transform, with the Gaussian first order derivative to find singularity exponents in the direct method, and the leaders coefficients for the multifractal formalism. The Legendre transform was used to estimate the multifractal spectrum, while the box-counting method was used to compute the Hausdorff dimension of sets of the same degree of singularity. Results demonstrate that it is more interesting in some cases to use the box-counting method than the Legendre transform to obtain a more accurate spectrum, as in the bimodal spectrum case.
\end{abstract}

Index Terms-Box-counting method, multifractal formalism, multifractal spectrum, texture classification.

\section{INTRODUCTION}

The Multifractal analysis and its formalism were designed initially to study the variations of flow velocity and especially to study the phenomenon of intermittent fully developed turbulence. Indeed, it is often related to objects of very complex geometry, anisotropic, inhomogeneous, very irregular texture and roughness that varies from one point to another, and that fractal analysis is not enough to give a fully description. Multifractal analysis is applied in dynamic system, in hydrology to model the rainfall, to study earthquakes, stock market, to study the distribution of matter in the universe, to model the Internet traffic, and also to study DNA sequences [1]-[3].

The basic principle of multifractal analysis is to compute the local dimension and consider the sets of the same degree of singularity to estimate the dimension of their distribution. The direct application of this method is difficult due to the high density of sets of the same exponents in most natural images. This constraint brought Frish and Parisi [4] to introduce the multifractal formalism. In this paper we propose to analyze textures with and without the use of this

Manuscript received April 5, 2014; revised June 5, 2014.

Khaled Harrar is with the Engineering Faculty, University of M'Hamed Bougara Boumerdes, Avenue de l'indépendance, 35000, Boumerdes, Algeria (e-mail: harrar k@umbb.dz)

Mohamed Khider is with the LTIR laboratory, Electrical engineering faculty, University of Science and Technology Houari Boumediene, BP 32, Bab Ezzouar, Algeria (e-mail: mohamed.khider@ieee.org) formalism. In addition, we propose to use the box-counting method to estimate the fractal dimension $D\left(h_{0} \pm \Delta h / 2\right)$ of sets with singularity exponent that satisfy the inequality $h_{0}-\Delta h / 2$ $<h_{x}<h_{0}+\Delta h / 2$, where $h_{0}$ is the Holder exponent of the middle interval considered, $h x$ is some Holder exponent belonging to the interval $\left[h_{0}-\Delta h / 2, h_{0}+\Delta h / 2\right]$, and $\Delta h$ indicates the interval size of the Holder set.

\section{MULTIFRACTAL SPECTRUM}

The Multifractal analysis considers the distribution of measurement $\mu(M(X, \sigma)) \sim \sigma^{h(X)}$, where $\mu$ denotes the measurement function in the sense of calculation, $M$ is the image or surface of grayscale, $X$ is a given position of a pixel in the image, $\sigma$ is the scale of analysis or the wavelet support and $h(X)$ is the Holder exponent, also called singularity. The multifractal spectrum gives an estimation of the Hausdorff fractal dimension $D(h)$ of the spatial distribution in the image sets (in the geometric sense, their positions in the image) of the same degree of singularity $h(x)$. The multifractal analysis is used to describe the fluctuations of the local regularity of a signal $f(x)$ represented by the Holder exponents also called local Hurst. The higher the value of $h\left(x_{0}\right)$, the greater the signal $f$ is regular and vice versa (more irregular). The information on the variability of the regularity of $f$ is described by the multifractal spectrum $D(h)$, which is the Hausdorff dimension of all points $x$ where the signal $f$ got the same Holder exponent $h$ [5]:

$$
D(h)=\operatorname{dim}_{h}\{x: h(x)=h\}
$$

To estimate this spectrum, we propose to compare two methods: (a) the multifractal formalism of Frish and Parisi and leaders coefficients, so by Legendre transform [4]; and (b) the direct method that uses the box-counting method to compute the Hausdorff dimension and uses modulus of continuous wavelet transform to estimate the singularity exponents for each pixel of the image.

\section{WAVELET TRANSFORM FOR MULTIFRACTAL ANALYSIS}

The use of the Wavelet Transform for Multifractal Measure (WTMM) was addressed in 1992 by the theory of Mallat and Hwang [6]. The introduction of methods such as the maximum modulus of the wavelet transform in WTMM for multifractal analysis of rough surfaces is due to works of Arnéodo et al., [7], and more recently Abry and Jaffard [8] have introduced the leaders coefficients with the discrete wavelet transform to find the multifractal spectrum.

The multifractal analysis based on leaders coefficients attract great interest due to gain in time computation and 
quality of results that can be achieved. This method is based on a theoretical foundation well established by Abry et al. [8] and Jaffard et al. [9]. The leaders coefficients are calculated from moduli of the Discrete Wavelet Transform (DWT). This explains the rapidity of the method, and the results are conclusive even using wavelet of lower order, e.g. Daubechies first order.

The multifractal spectrum method of the leaders coefficients is obtained using the notation of Lashermes [10]. In the one dimensional case for example, for each dyadic interval at scale $2^{j}, d_{f}(j, k)$ denotes the coefficient of the DWT. The leaders coefficient $l_{f}(j, k)$ are given by [10]:

$$
l_{f}(j, k)=\sup _{\lambda^{\prime} \subset 3 \lambda(j, k), j^{\prime} \leq j}\left|d_{f}\left(\lambda^{\prime}\right)\right|
$$

We use the following partition functions, taking $d=2$ (2D case):

$$
S_{f}^{l}(q, j)=\frac{1}{n(j)} \sum k_{i} \frac{1}{2^{d}-1} \sum_{m}\left(l_{f}\left(j, k_{i}, m\right)\right)^{q}
$$

We denote by $q$ the moment, $n(j)$ the number of leaders coefficients at the octave $j$. The scale function $\zeta_{f}^{l}(q)$ is derived from the relation $\lim _{j \rightarrow 0} \inf \frac{\ln S_{f}^{l}(q, j)}{\ln 2^{j}}=\zeta_{f}^{l}(q)$, and with $D(h)=\operatorname{dim}_{h}\{x: h(x)=h\}$

Legendre transform :

$$
D_{f}^{l}(h)=\min _{q}\left(d+q h-\zeta_{f}^{l}(q)\right)
$$

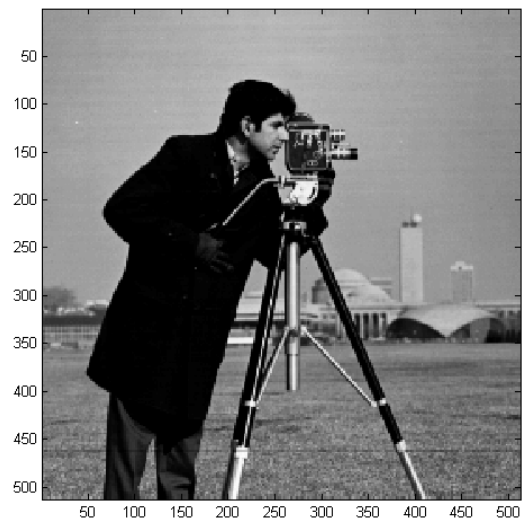

Fig. 1. A grayscale original image.

This relation is called multifractal formalism of Frish and Parisi. It makes the estimation of the multifractal spectrum possible through the Legendre transform.

In this work, we propose to analyze an image with the box-counting method and Legendre multifractal spectrum by the Leader coefficients method. Fig. 1 illustrates a $512 \times 512$ natural grayscale image encoded on 8 bits. Fig. 2 presents a convex scaling function of the original image, where we can notice a multifractal character. Fig. 3 represents the multifractal spectrum obtained with Legendre transform while using the leader wavelets coefficients, a convexity is noticed in the spectrum. Fig. 4 illustrates the partition functions for -5 to +5 statistical moments, these results are obtained using the first order Daubechies wavelets.

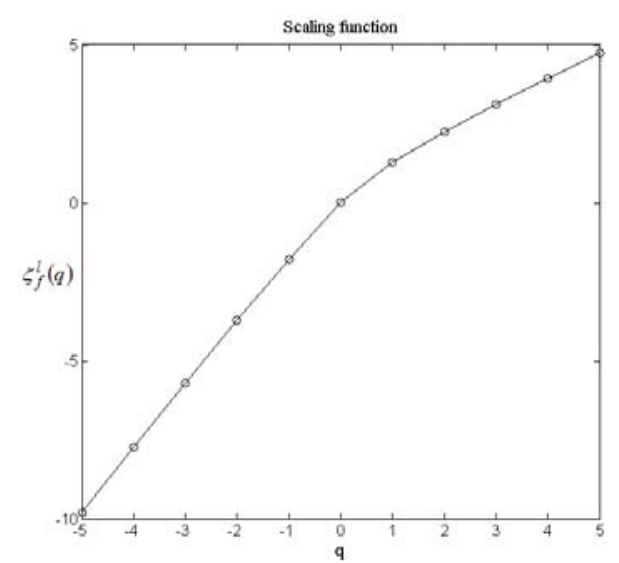

Fig. 2. The scaling function for the moments $-5<q<+5$.

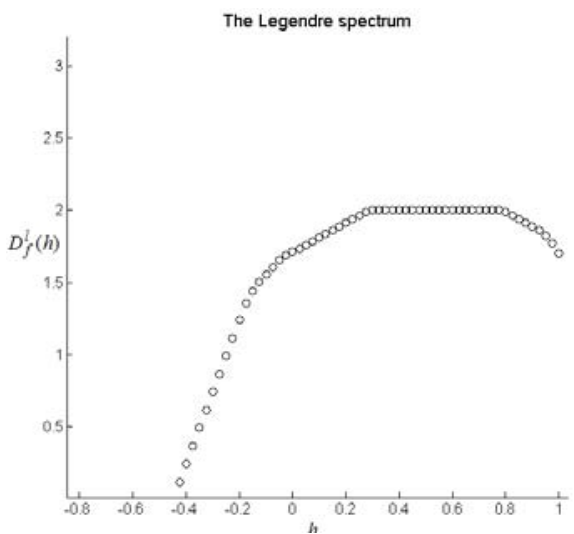

Fig. 3. The Legendre multifractal spectrum by the leaders coefficients method.

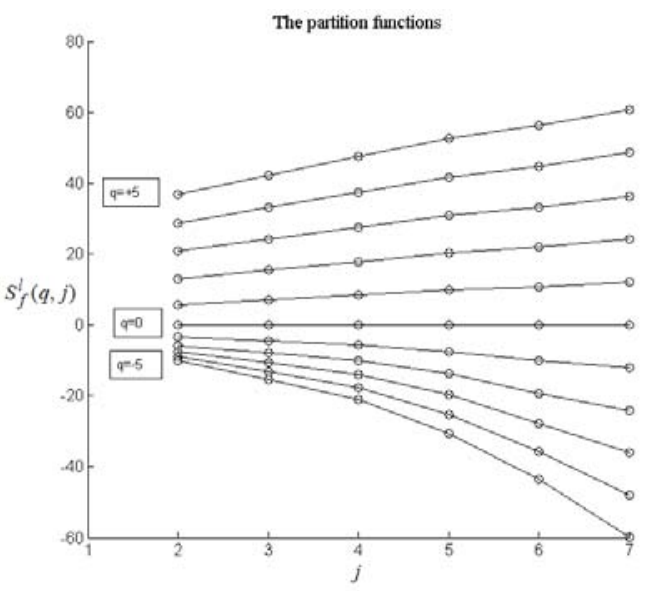

Fig. 4. Log-Log of the partition functions.

\section{Multifractal Analysis By BoX-Counting Method}

One of the most famous empirical algorithms for calculating the fractal dimension is the "box-counting" method [11]-[13]. In order to find the Hausdorff dimension, intervals $\left[h_{1}, h_{2}\right]$ of fixed size $\Delta h=h_{2}-h_{1}$ are used to estimate the fractal dimension of the set belonging to the interval $\left[h_{1}\right.$, $h_{2}$ ] which is given by the notation $D\left(h_{1} / 2+h_{2} / 2\right)$. This dimension is obtained from the slope by fitting the linear regression of the number of boxes versus scales of analysis in a Log-Log plot [11]:

$$
\operatorname{dim}_{B}(F)=\lim _{\sigma \rightarrow 0+}\left[\frac{\log N_{0}(F)}{- \text { long }}\right]
$$


To estimate the Hausdorff dimension, pixels with Holder exponents belonging to the interval $\left[h_{1}, h_{2}\right]$ are set to " 1 " and the others to " 0 ". The box dimension is computed by the box-counting method of the obtained binary image. Different obtained results using the multifractal analysis of the direct method are presented. Fig. 5 gives the histogram of these Holder exponents, this result is deduced by analyzing the local variation of moduli of continuous wavelets transform. Fig. 6 represents the predominant Holder set of the image; it shows the spatial distribution of the sets that form the spectrum mode. Fig. 7 shows the most singular Holder exponent set of the image, these sets are focused in edges of the image. Figure 8 illustrates the multifractal spectrum estimation by the direct method without Legendre transform. To do so, the box-counting algorithm is used and the fractal dimension is calculated for several $\Delta h$ values. In Fig. 9, the color map of Holder exponent for the previous image is given. Finally, Fig. 10 presents the Legendre spectrum, in bleu the most singular set, and in red the Legendre spectrum mode.

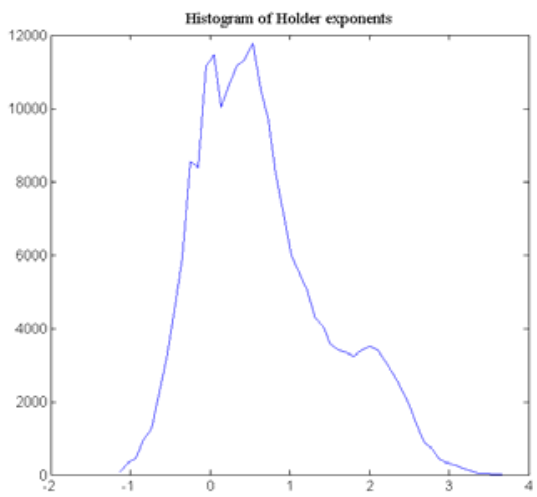

Fig. 5. Histogram of singularity exponents.

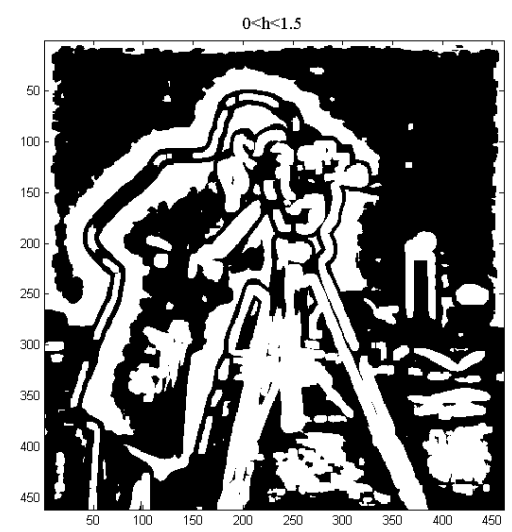

Fig. 6. The set of predominant multifractal coinciding with the peak in the spectrum mode.

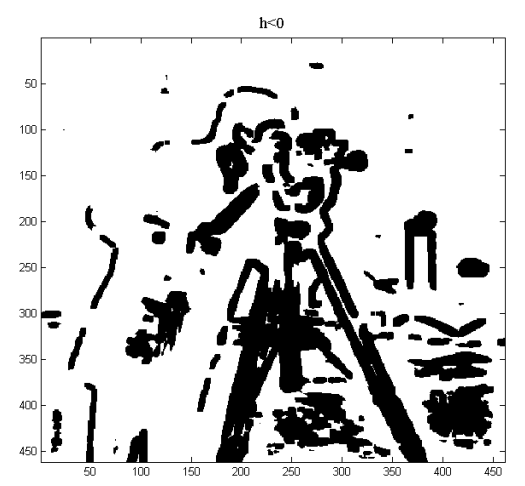

Fig. 7. The most singular Holder exponent set of the image.

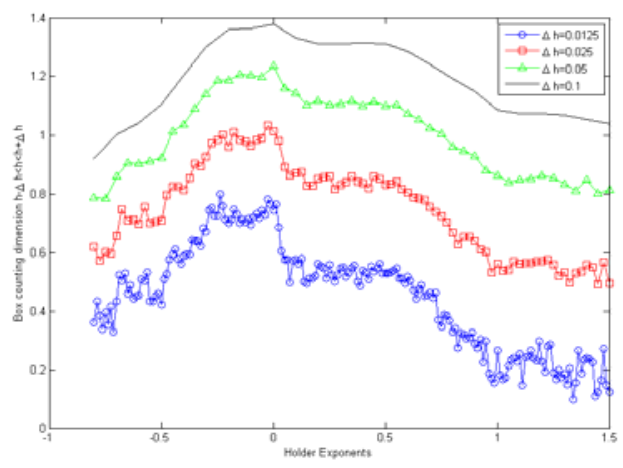

Fig. 8. Multifractal spectrum estimation by the box-counting method.

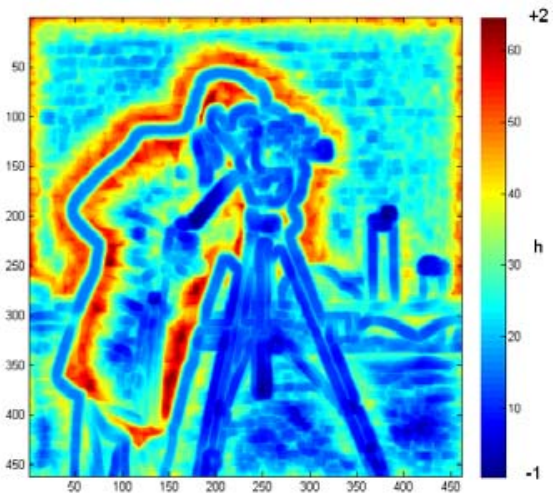

Fig. 9. Holder exponents in color map.

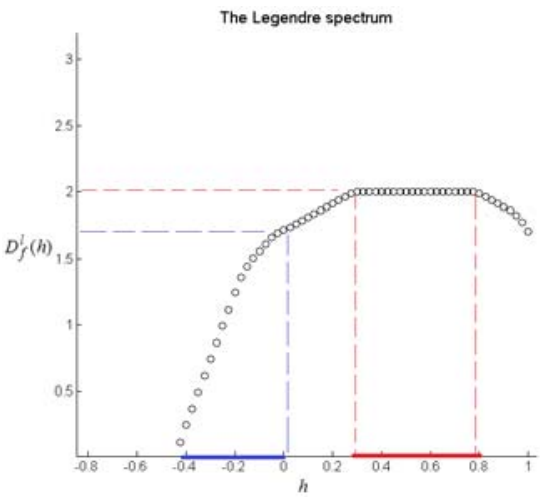

Fig. 10. Legendre spectrum indicating the predominant set and the most singular Holder exponents.

\section{Classification Using Multifractal Spectrum MODE: RESULTS AND DISCUSSION}

Robust classification methods are based on the use of the mode of the multifractal spectrum, called predominant Holder exponent, which represents the peak of the spectrum singularity. In image processing, multifractal analysis has revealed that different textures have different exponent singularities. Several authors have demonstrated the effectiveness of this procedure in classification of images [14]-[16]. In this paper, based on: -the histogram of the singularity exponents, -the Legendre multifractal spectrum obtained after applying the multifractal formalism and -the spectrum estimated with the box-counting method and the continuous wavelet transform, we propose to classify the Brodatz texture database. Tables I, II and III, illustrate some results which show the three parameters of spectra and histogram $h_{\min }, h_{\max }$ and $h_{p i c}$ for the first 16 Brodatz textures (Fig. 11) [17]. In this paper, we have used natural textures 
which have in the most case a power spectrum of radial frequency $f$ whose amplitude follows a power law of the type: : $S(f)=a l f^{b}$. Some authors have even suggested an average value of 1.08 for $b$ [18]. We find this type of process $1 / f$, for example in the case of Brownian surfaces. This power spectrum exponent is related to the Hurst exponent by the relation $b=2 H+2$. So it is interesting to characterize such textures using multifractal analysis.

In Table I, the parameters $h_{\min }, h_{\max }$ and $h_{p i c}$ indicate respectively the singularity exponents of Brodatz textures, the minimum, the maximum and the Holder exponent of the spectrum mode with $D\left(h_{\min }\right)=D\left(h_{\max }\right)=0$ and $D\left(h_{p i c}\right)=\max$ $(D(h))$, with $h$ belonging to $\left[h_{\min }, h_{\max }\right]$, it is possible to detect the monofractal character, denoted by $\mathrm{M}$ in the table.

The Table II presents the results obtained with the box-counting method to calculate the fractal dimension of the spectrum. We notice that it is possible to obtain the multimodal spectra that cannot be obtained by the Legendre transform method. It also appears that it is difficult to detect the monofractal character of certain textures. In this case it is possible to use the multifractality index $D h_{\operatorname{multi}}=h_{\max }-h_{\min }$.

This parameter is less important in the case of monofractal textures.

In Table III, the parameters $h_{\min }, h_{\max }$ and $h_{p i c}$ are obtained directly from the histogram of the singularity exponents through the study of the moduli of continuous wavelets transform evolution in each pixel of the image.

To highlight the bimodal character, others Brodatz textures $\left(D_{101}, D_{102}, D_{4}, D_{57}, D_{62}\right.$ and $\left.D_{87}\right)$ presented in Fig. 11 were analyzed. As can be seen on Fig. 12, the obtained results with and without the Legendre transform reveal differences texture analysis. A significant difference between the peaks of the spectra is observed for Brodatz textures $D_{102}$ and $D_{101}$. In fact, for these textures, the spectrum estimated by the direct method reflects a bimodal character; this could not be seen with the multifractal formalism of Frisch and Parisi, which uses a convex scaling function. It is interesting to study the shape of the multifractal spectrum, which is calculated with the box-counting method on $\Delta h$ in a predefined intervals in order to obtain $D\left(h_{p i c}\right)$ close to the value 2, which means that the predominant Holder set pave the image as a surface.

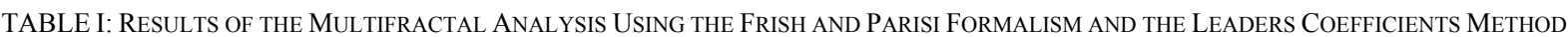

\begin{tabular}{|c|c|c|c|c|c|c|c|c|c|c|c|c|c|c|c|}
\hline Texture & $D_{1}$ & $D_{2}$ & $D_{3}$ & $D_{4}$ & $D_{5}$ & $D_{6}$ & $D_{7}$ & $D_{8}$ & $D_{9}$ & $D_{10}$ & $D_{11}$ & $D_{12}$ & $D_{13}$ & $D_{15}$ & $D_{16}$ \\
\hline$h_{\min }$ & -0.87 & -0.48 & -0.9 & $M$ & -0.5 & -1.17 & -0.4 & -0.3 & $M$ & -0.8 & $M$ & -0.5 & -0.47 & -0.83 & $M$ \\
\hline$\overline{h_{\max }}$ & 0.45 & 0.8 & 0.22 & $M$ & 0.6 & -0.05 & 0.97 & 0.65 & $M$ & 0.7 & $M$ & 0.7 & 0.98 & 0.38 & $M$ \\
\hline $\boldsymbol{h}_{\text {pic }}$ & -0.23 & 0.02 & -0.5 & -0.5 & -0.1 & -0.7 & 0.2 & 0.175 & -0.5 & -0.18 & -0.15 & -0.15 & 0.07 & -0.38 & -0.65 \\
\hline$D\left(h_{\text {pic }}\right)$ & 2 & 2 & 2 & 2 & 2 & 2 & 2 & 2 & 2 & 2 & 2 & 2 & 2 & 2 & 2 \\
\hline
\end{tabular}

TABLE II: RESULTS OF THE MULTIFRACTAL ANALYSIS USING THE BOX-COUNTING METHOD APPLIED TO BRODATZ TEXTURE
\begin{tabular}{|l|l|l|l|l|l|l|l|l|l|l|l|l|l|l|l|}
\hline Texture & $\boldsymbol{D}_{\mathbf{1}}$ & $\boldsymbol{D}_{\mathbf{2}}$ & $\boldsymbol{D}_{\mathbf{3}}$ & $\boldsymbol{D}_{\mathbf{4}}$ & $\boldsymbol{D}_{\mathbf{5}}$ & $\boldsymbol{D}_{\mathbf{6}}$ & $\boldsymbol{D}_{\mathbf{7}}$ & $\boldsymbol{D}_{\mathbf{8}}$ & $\boldsymbol{D}_{\mathbf{9}}$ & $\boldsymbol{D}_{\mathbf{1 0}}$ & $\boldsymbol{D}_{\mathbf{1 1}}$ & $\boldsymbol{D}_{\mathbf{1 2}}$ & $\boldsymbol{D}_{\mathbf{1 3}}$ & $\boldsymbol{D}_{\mathbf{1 5}}$ & $\boldsymbol{D}_{\mathbf{1 6}}$ \\
\hline $\boldsymbol{h}_{\min }$ & $<-2$ & -1.7 & $<-2$ & $<-2$ & -1.8 & $<-2$ & -1.7 & -1.4 & -2 & -2 & -1.7 & -1.9 & -1.7 & -1.9 & $<-2$ \\
\hline $\boldsymbol{h}_{\max }$ & 1.2 & 1.8 & 1.1 & 0.47 & 1.4 & 0.7 & $>2$ & 1.4 & 0.6 & 1.5 & 1 & 1.6 & $>2$ & 1.1 & 0.4 \\
\hline $\boldsymbol{h}_{\boldsymbol{p} \text { ic }}$ & 0.1 & 0 & -0.7 & -0.7 & -0.1 & -0.7 & 0.3 & 0.2 & -0.5 & -0.4 & -0.1 & -0.2 & 0.1 & -0.4 & -0.7 \\
\hline $\boldsymbol{D}\left(\boldsymbol{h}_{\boldsymbol{p} \text { ic }}\right)$ & 1.77 & 1.84 & 1.84 & 1.87 & 1.84 & 1.84 & 1.74 & 1.86 & 1.88 & 1.75 & 1.88 & 1.84 & 1.79 & 1.85 & 1.88 \\
\hline
\end{tabular}

TABLE III: SINGULARITY EXPONENTS FOLLOWING THE HISTOGRAM WITHOUT CALCULATING THE FRACTAL DIMENSION

\begin{tabular}{|l|l|l|l|l|l|l|l|l|l|l|l|l|l|l|l|}
\hline Texture & $\boldsymbol{D}_{\mathbf{1}}$ & $\boldsymbol{D}_{\mathbf{2}}$ & $\boldsymbol{D}_{\mathbf{3}}$ & $\boldsymbol{D}_{\mathbf{4}}$ & $\boldsymbol{D}_{\mathbf{5}}$ & $\boldsymbol{D}_{\mathbf{6}}$ & $\boldsymbol{D}_{\mathbf{7}}$ & $\boldsymbol{D}_{\mathbf{8}}$ & $\boldsymbol{D}_{\mathbf{9}}$ & $\boldsymbol{D}_{\mathbf{1 0}}$ & $\boldsymbol{D}_{\mathbf{1 1}}$ & $\boldsymbol{D}_{\mathbf{1 2}}$ & $\boldsymbol{D}_{\mathbf{1 3}}$ & $\boldsymbol{D}_{\mathbf{1 5}}$ & $\boldsymbol{D}_{\mathbf{1 6}}$ \\
\hline $\boldsymbol{h}_{\text {min }}$ & -2 & -1.33 & -2 & -2 & -1.5 & -2.27 & -1.2 & -1 & 0.6 & 1.5 & 1 & 1.6 & $>2$ & 1.1 & 0.4 \\
\hline $\boldsymbol{h}_{\text {max }}$ & +2 & 1.82 & 1.5 & 1 & 1.5 & 1.62 & 2.23 & 1.5 & -0.5 & -0.4 & -0.1 & -0.2 & 0.1 & -0.4 & -0.7 \\
\hline $\boldsymbol{h}_{\text {pic }}$ & 0.113 & -0.095 & -0.69 & -0.63 & -0.08 & -0.713 & 0.07 & 0.22 & -2 & -2 & -1.7 & -1.9 & -1.7 & -1.9 & $<-2$ \\
\hline
\end{tabular}

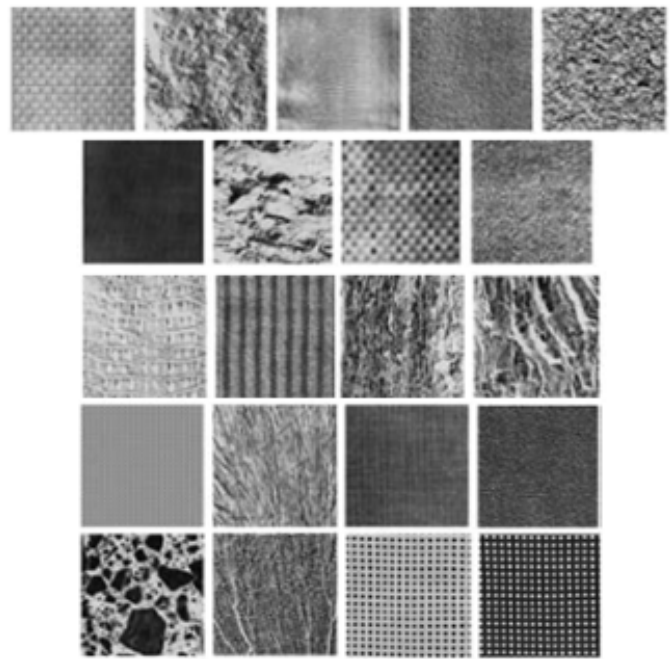

Fig. 11. Brodatz texture set. From left to right and top to bottom: $D_{1}, D_{2}, \ldots$, $D_{16} . D_{57}, D_{62}, D_{87}, D_{101}, D 102$.
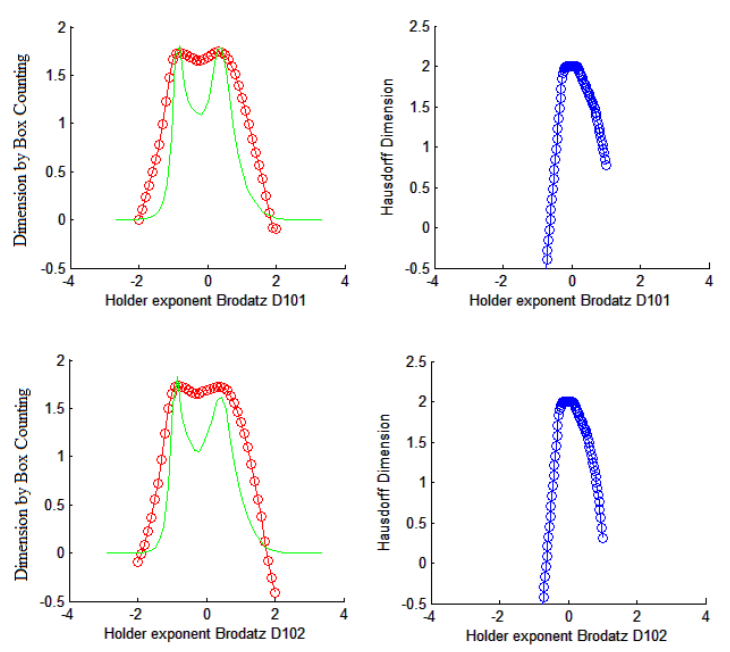

Fig. 12. Bimodal multifractal spectrum obtained using the box-counting method (red color). An adjusted histogram of holder exponent (green color). The multifractal spectrum obtained with leaders wavelets method (blue color). 

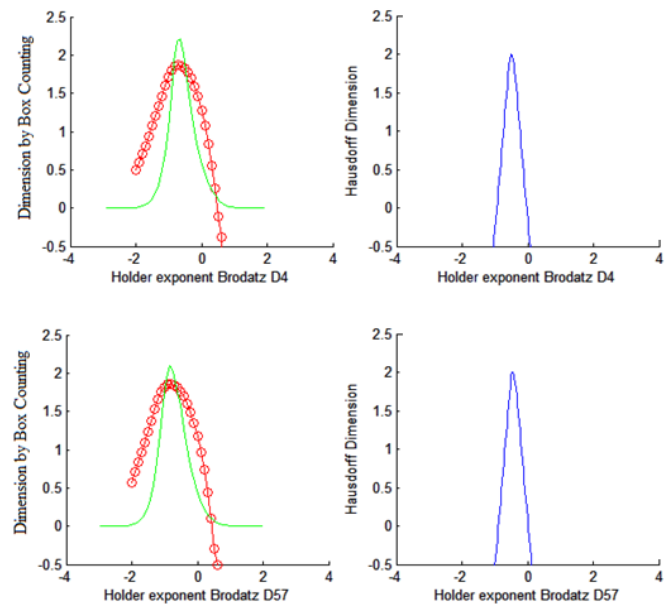

Fig. 13. Monofractal spectrum obtained using the box-counting method (red color).An adjusted histogram of holder exponent (green color).The multifractal spectrum obtained with leaders wavelets method (blue color). In this last case the spectrum is reduced to an intersection of two segments.
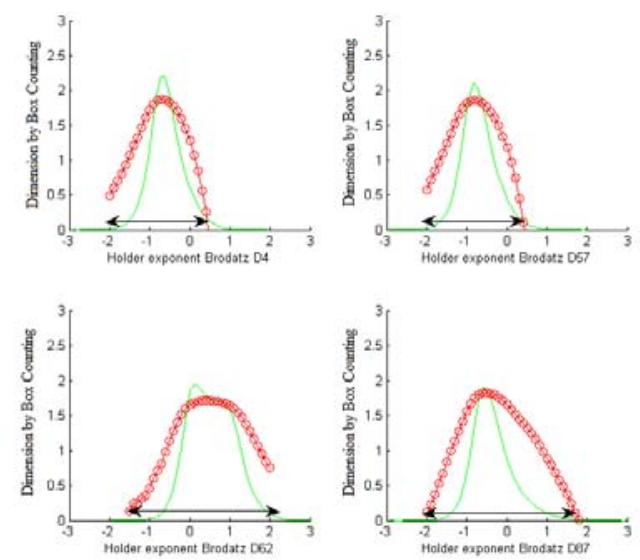

Fig. 14. The multifractal and monofractal cases can be detected with the multifractality index $h_{\max }-h_{\min }$ as indicated in this figure. The Brodatz texture D62 and D87 reveal a multifractal character, when $D_{4}$ and $D_{57}$ reveal a monofractal character with less important $h_{\max }-h_{\min }$. The histogram of exponents singularities is given, the later is suitably adjusted to match the multifractal spectrum.

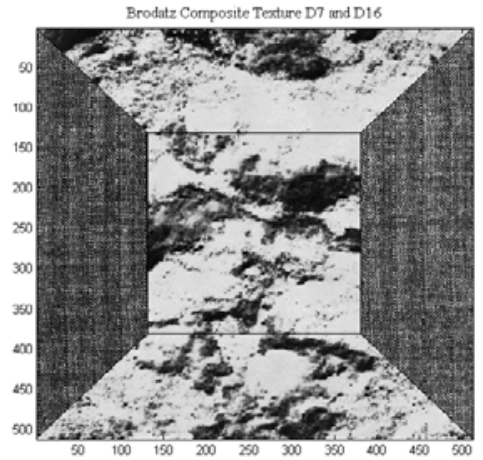

Fig. 15. Composite image, obtained by combining two Brodatz textures D7 and $D_{16}$.

Moreover, we notice (Fig. 13 and Fig. 14) that significant differences are observed in the multifractal measures for textures that represent the least homogeneity e.g. $D_{62}$ and D87. The comparison is made between the multifractal spectrum and the modes of the histogram of singularity exponents; this can be explained by the fact that the largest set of the histogram doesn't necessarily represent the peak of the multifractal spectrum. In addition, the index of multifractality which also indicate the support of the multifractal spectrum given by $h_{\max }-h_{\min }$, can be used to reveal the monofractal nature with the direct method. Indeed, the support of the spectrum is less important in the monofractal case, as shown in the results of calculation in Brodatz textures $D_{4}$ and $D_{57}$.

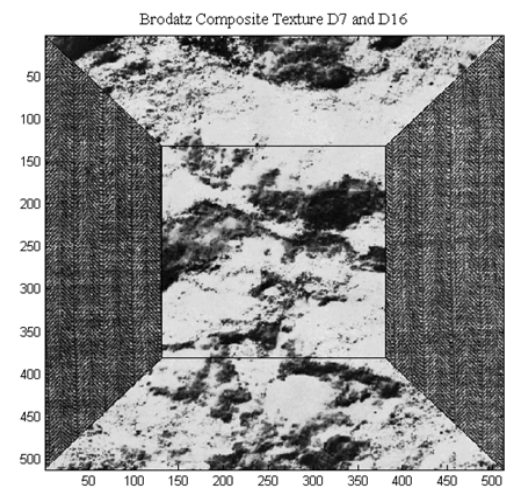

Fig. 15. Composite image, obtained by combining two Brodatz textures $D_{7}$ and $D_{16}$.

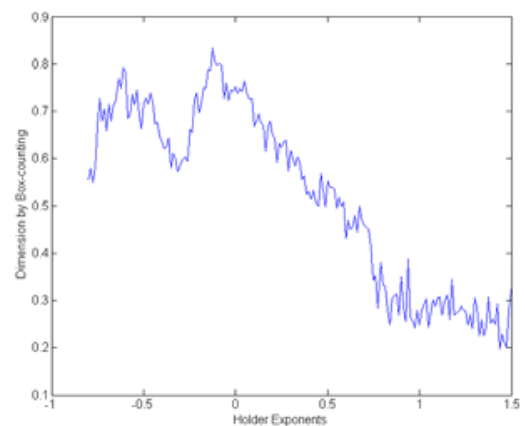

Fig. 16. Multifractal spectrum of the previous composite texture, the fractal dimension is calculated with box-counting method for $\Delta h=0.0125$, a bimodal character of the multifractal spectrum is observed.

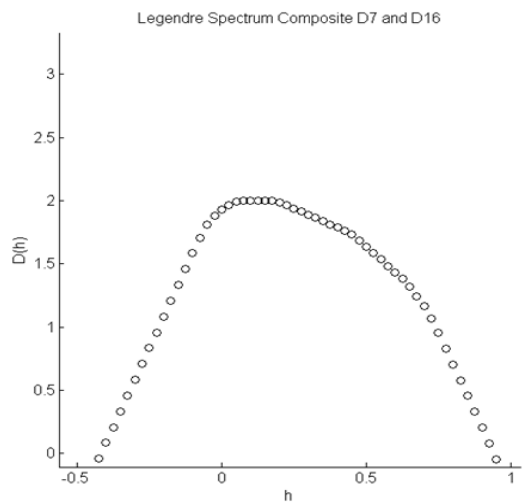

Fig. 17. Multifractal analysis with Leader coefficients method, this result does not reflect the nature of the composite texture.

In this study we propose also to analyze a composite image, generated using two Brodatz textures $D_{7}$ and $D_{16}$. In fact, the modes of the spectra of the two textures are quite distinct, as shown in the results table of the multifractal analysis of Brodatz textures. The use of the method based on multifractal formalism and the Legendre transform with the dominant coefficients does not reflect the composite nature of the analyzed image presented in Fig. 15, since the spectrum show only a single mode (Fig. 17). On the contrary, using the proposed method based on a box-counting approach with $\Delta h$ $=0.0125$, allows the observation of the bimodal spectrum, due to the composite nature of the texture image (Fig. 16). 


\section{CONCLUSION}

This study allowed us to compare the results of multifractal analysis using the direct method based on the box-counting to estimate the Hausdorff dimension of sets whose Holder exponent $\mathrm{h}_{\mathrm{x}}$ satisfies the relation $h_{0}-\Delta h / 2<h_{x}$ $<h_{0}+\Delta h / 2$, and the method based on the multifractal formalism of Frisch and Parisi through leaders coefficients of the discrete wavelets transform. Although the latter is faster and requires less computation time, it cannot reveal the bimodal character observed for some textures.

In conclusion, it is possible to detect the monofractal character of some textures by applying the Legendre transform. The multimodal nature that characterizes some textures is more difficult to emphasize, this can be done using the box-counting method. Thus, it is interesting to combine these two techniques for more efficient analysis.

\section{REFERENCES}

[1] P. Hubert, Y. Tessier et al., "Multifractals and extreme rainfall events," Geophys. Res. Lett., vol. 20, pp. 931-934, 1993.

[2] B. J. T. Jones, V. J. Martinez, E. Saar, and J. Einasto, "Multifractal description of the large-scale structure of the universe," Astrophys. J., vol. 332, pp. L1-L5, 1988.

[3] T. Hirabayashi, K. Ito, and T. Yoshii, "Multifractal analysis of earthquakes," Pure App. Geophys., vol. 138, no. 4, pp. 591-610, 1992.

[4] U. Frisch and G. Parisi, "Turbulence and predictability in geophysical fluid and climate dynamics," Elsevier, North Holand New York, pp. 84-88, 1995.

[5] P. Kestener, "Analyse multifractale 2D et $3 \mathrm{D}$ à l'aide de la transformation en ondelettes: Application en mammographie et en turbulence développée," PhD. dissertation, Univ. Bordeaux I, 2003.

[6] S. Mallat and W. L. Hwang, "Singularity detection and processing with wavelets," IEEE Trans. Inform. Theory, vol. 38, no. 2. pp. 617-643, 1992.

[7] A. Arneodo, E. Bacry, and J. F. Muzy, "The thermodynamics of fractals revisited with wavelets," Physica A - Stat. Mech. App., vol. 213, pp. 232-275, 1995

[8] P. Abry, S. Jaffard, and B. Lashermes, "Revisiting scaling, multifractal, and multiplicative cascades with the wavelet leaders lens," in Proc. of SPIE, 2004, vol. 5607, pp. 103-117.

[9] S. Jaffard, B. Lashermes, and P. Abry, "Wavelet in multifractal analysis," in Wavelet Analysis and Applications, Birkhauser Verlag, Basel, Switzerland, 2006, pp. 219-264.

[10] B. Lashermes, "Analyse multifractale pratique: Coefficients dominants et ordres critiques. Applications à la turbulence pleinement développée. effets de nombre de Reynolds fini," Ph.D. dissertation, High Normal School, Lyon, 2005.

[11] K. Falconer, Fractal Geometry: Mathematical Foundations and Applications, 2nd ed., John Wiley and Sons, 2003, pp. 328.
[12] K. Harrar and L. Hamami, "Implementation of the box-counting method in radiographic images," Advances in Numerical Methods, Springer Science+Business Media, 2009, pp. 299-311.

[13] K. Harrar and L. Hamami, "An interconnectivity index for osteoporosis assessment in x-rays images," J. Med. Biol. Eng., vol. 33, no. 6, pp. 569-575, 2013.

[14] M. Khider, A. Taleb-Ahmed, Abdelmalik, P. Dubois, and B. Haddad, "Classification of trabecular bone texture from MRI and CT scan images by multi resolution analysis," in Proc. 2007b IEEE Eng Med Biol Soc conf., 2007, pp. 5589-5592.

[15] M. Khider, B. Haddad, and A. Taleb-Ahmed, "Analyse multi fractale des échos radar par la méthode Maxima du Module de la Transformée en ondelettes (MMTO) 2D pour les sites de Bordeaux (France), Sétif (Algérie) : Application à l'élimination des échos parasites," Rev. Tel., vol. 8, no. 4, pp. 271-283, 2009.

[16] M. Khider, A. Taleb-Ahmed, and B. Haddad, "Generation of synthetic multifractal realistic surfaces based on natural model and lognormal cascade: Application to MRI classification," LNCS, Springer-Verlag. pp. 71-78, 2010.

[17] P. Brodatz, Texture: A Photographic Album for Artists and Designers, Dover Publications, Inc., New York, 1966.

[18] V. A. Billock and G. C Guzman, "Fractal time and 1/f spectra in dynamic images and human vision," Physica D, vol. 148, pp. 136-146, 2001 .

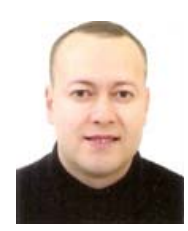

Khaled Harrar received his engineering degree in electronic in 1997 from University of science and technology Houari Boumediene (USTHB) of Algiers, Algeria. A magister diploma in electronic in 2001 from the USTHB, and in February 2014 he received his $\mathrm{PhD}$ degree from National polytechnical school (ENP) Algiers, Algeria. He is a member of LSC laboratory, and also a reviewer in an international journal. He is a professor assistant at M'hamed Bougara Boumerdes University since 2004. His research interest concerns signal and image processing including complex texture characterizing by fractal analysis, fractional Brownian motion models, and other approaches based on SVM and neural network technique, for classification, computer aided detection and diagnosis in medical applications. Dr. Harrar has made valuable contribution to research in bone texture characterization and has a number of publications to his credit in International Journals of high repute.

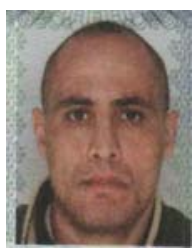

Mohamed Khider received his $\mathrm{PhD}$ degree from University of science and technology Houari Boumediene (USTHB), Algiers, Algeria (2011). He is an assistant professor and a member of LTIR laboratory, USTHB, and also an IEEE member since 2012. His field of interest includes signal processing for oil prospecting, by using Vibroseis technique, multifractal analysis of signals applied to medical and meteorological radar imaging, multiresolution analysis, generation of synthetic multifractal surfaces, Bayesian methods, and other approaches based on neural network technique for classification. Dr. Khider has several papers in national and international journals. 